\title{
Some exact correlations in the Dyson Brownian motion model for transitions to the CUE
}

\author{
P.J. Forrester* \\ Department of Mathematics \\ University of Melbourne \\ Parkville
}

Victoria 3052

Australia

\begin{abstract}
The Dyson Brownian motion model for transitions to the CUE is considered. For initial eigenvalue probability density functions corresponding to the COE and CSE, the density-density correlation function between an eigenvalue at position $\lambda^{\prime}$ initially $(\tau=0)$ and an eigenvalue at position $\lambda$ for general $\tau$, is calculated. Theoretical predictions for the asymptotic behaviour are verified, and the distribution for the initial rate of change of the eigenvalues is calculated. Also, for initial conditions corresponding to equi-spaced eigenvalues, the $n$-point equal parameter distribution is computed. The extension of the calculation of these quantities to a multiparameter version of the theory is given.
\end{abstract}

\section{Introduction}

A problem of fundamental importance in studying quantum aspects of classically chaotic systems is the statistical properties of the energy spectra. Since the pioneering work of Wigner and Dyson (see e.g. [1]) it has been realized that the statistical properties are determined by the presence, or not, of a global time reversal symmetry $T$. Details of the matrix elements of the Hamiltonian are unimportant, apart from the global constraint imposed by the symmetry $T$. Thus the discrete portion of the energy spectrum can be studied by modelling the Hamiltonian by a random Hermitian matrix if there is no time reversal symmetry, and in the case of a time reversal symmetry by a random real symmetric matrix if $T^{2}=1$ and a random real quaternion matrix if $T^{2}=-1$.

More complicated situations can arise. In particular the quantum system may contain a parameter which can continuously vary the spectrum within a universality class or indeed between universality classes (see e.g. $[2,3]$ ). For the latter situation, by considering parameter $(\tau)$ dependent Gaussian random matrices (see e.g. [4]), it is possible to show that the eigenvalue probability density function (p.d.f.)

*email: matpjf@maths.mu.oz.au; supported by the ARC 
$p\left(\lambda_{1}, \ldots, \lambda_{N} ; \tau\right)$ satisfies the Fokker-Planck equation

$$
\gamma \frac{\partial p}{\partial \tau}=\mathcal{L} p, \quad \mathcal{L}=\sum_{j=1}^{N} \frac{\partial}{\partial \lambda_{j}}\left(\frac{\partial W}{\partial \lambda_{j}}+\beta^{-1} \frac{\partial}{\partial \lambda_{j}}\right)
$$

where

$$
W=-\sum_{1 \leq j<k \leq N} \log \left|\lambda_{j}-\lambda_{k}\right|+\frac{1}{2} \sum_{j=1}^{N} \lambda_{j}^{2} .
$$

Here $\gamma$ provides a scale for $\tau / \lambda_{j}^{2}$ and $\beta=1,2$ or 4 depending on whether the transition is to orthogonal, unitary or symplectic symmetry. With $\tau=0, p$ is specified as the eigenvalue p.d.f. of one of the three Gaussian random matrix ensembles, with a symmetry different to that after the transition. In the random matrix context, the Fokker-Planck equation (1.1) is referred to as the Dyson Brownian motion model.

The objective of this paper is to provide the exact evaluation of some two-point correlation functions for the transition to unitary symmetry. In Section 2 we revise exact results on this problem which are already known $[5,6]$, as well as a theoretical prediction for asymptotic behaviours, the implication this prediction has for the integrated correlator of the variance of a linear statistic [7], and the calculation of the distribution function for the initial rate of change of the eigenvalues. In Section 3 we provide the exact evaluation for the density-density correlation between an eigenvalue at $\tau=0$ and an eigenvalue at general $\tau$, when the eigenvalue p.d.f at $\tau=0$ corresponds to orthogonal symmetry, while in Section 4 the calculation will be repeated with the $\tau=0$ eigenvalue p.d.f. corresponding to symplectic symmetry. In both cases the expected asymptotic behaviour is verified, and the distribution function for the initial rate of change of the eigenvalues is calculated. In Section 5 we calculate the equal-parameter $n$-point correlations when the initial conditions correspond to equispaced eigenvalues, while in Section 6 we show how the results of the previous sections can be extended to apply to the multiple parameter case.

\section{Summary of known results}

\subsection{Equal parameter distributions}

The so called equal-parameter distribution functions

$$
\rho_{n}\left(\lambda_{1}, \ldots, \lambda_{N} ; \tau\right):=N(N-1) \ldots(N-n)\left(\prod_{l=n+1}^{N} \int_{-\infty}^{\infty} d \lambda_{l}\right) p\left(\lambda_{1}, \ldots, \lambda_{N} ; \tau\right)
$$

have been calculated exactly for the Fokker-Planck equation (1.1) with $\beta=2$, and initial conditions

$$
\begin{gathered}
p\left(\lambda_{1}, \ldots, \lambda_{N} ; 0\right)=\frac{1}{C_{1 N}} e^{-W\left(\lambda_{1}, \ldots, \lambda_{N}\right)} \\
p\left(\lambda_{1}, \ldots, \lambda_{N} ; 0\right)=\frac{1}{C_{4(N / 2)}} e^{-4 W\left(\lambda_{2}, \lambda_{4}, \ldots, \lambda_{N}\right)} \prod_{l=1}^{N / 2} \delta\left(\lambda_{2 l}-\lambda_{2 l-1}\right) \quad(N \text { even })
\end{gathered}
$$

in refs. $[5,6]$.

We are particularly interested in these distribution functions in the thermodynamic limit, when $N \rightarrow$ $\infty$ while the density of eigenvalues is fixed at some non-zero constant $\rho$. In the $N \rightarrow \infty$ limit, the density implied by (1.1) is (see e.g. [4]) the Wigner semi-circle

$$
\rho(\lambda) \sim \frac{2 \sqrt{2 N}}{\pi} \sqrt{1-\lambda^{2} / 2 N}
$$


so to obtain a bulk density $\rho$ about the origin it is necessary to scale the eigenvalues $\lambda_{j} \mapsto \pi \rho \lambda_{j} / \sqrt{2 N}$. This can be achieved by instead scaling $\gamma$ so that

$$
\gamma \mapsto 2 N \gamma /(\pi \rho)^{2}
$$

With this scale, the thermodynamic limit of the equal time distributions (2.1) exist and can be computed exactly for the initial conditions (2.2). In particular, for the initial conditions (2.2), the two-point distribution function is given by $[5,6]$ (for consistency with our notation the parameter $\rho^{2}$ in [5] is identified with $\rho^{2} \tau / 2 \gamma$ and $r$ replaced by $\rho \lambda$ )

$$
\begin{aligned}
\rho_{(2)}\left(\lambda_{1}, \lambda_{2}\right) & =\rho^{2}-\frac{\rho^{2}}{2} \operatorname{Tr}\left[\begin{array}{cc}
a(\lambda) & a_{12}^{\beta_{0}}(\lambda, \tau) \\
a_{21}^{\beta_{0}}(\lambda, \tau) & a(\lambda)
\end{array}\right]\left[\begin{array}{cc}
a(\lambda) & -a_{12}^{\beta_{0}}(\lambda, \tau) \\
-a_{21}^{\beta_{0}}(\lambda, \tau) & a(\lambda)
\end{array}\right] \\
& =\rho^{2}-\rho^{2}\left(a(\lambda)^{2}-a_{12}^{\beta_{0}}(\lambda, \tau) a_{21}^{\beta_{0}}(\lambda, \tau)\right)
\end{aligned}
$$

where

$$
\lambda:=\lambda_{1}-\lambda_{2} \quad a(\lambda):=\frac{\sin \pi \rho \lambda}{\pi \rho \lambda}
$$

and for the initial condition $(2.2 \mathrm{a})\left(\beta_{0}=1\right)$

$$
\begin{gathered}
a_{12}^{1}(\lambda, \tau):=-\pi \int_{0}^{1} d u u \sin \pi u \rho \lambda e^{\pi^{2} \rho^{2} \tau u^{2} / \gamma} \\
a_{21}^{1}(\lambda, \tau):=-\frac{1}{\pi} \int_{1}^{\infty} d u \frac{\sin \pi u \rho \lambda}{u} e^{-\pi^{2} \rho^{2} \tau u^{2} / \gamma},
\end{gathered}
$$

while for the initial condition $(2.2 \mathrm{~b})$

$$
\begin{gathered}
a_{12}^{4}(\lambda, \tau):=-\pi \int_{1}^{\infty} d u u \sin \pi u \rho \lambda e^{-\pi^{2} \rho^{2} \tau u^{2} / \gamma} \\
a_{21}^{4}(\lambda, \tau):=-\frac{1}{\pi} \int_{0}^{1} d u \frac{\sin \pi u \rho \lambda}{u} e^{\pi^{2} \rho^{2} \tau u^{2} / \gamma} .
\end{gathered}
$$

\subsection{Density-density and current-current correlations}

To define the density-density correlation, $S\left(\lambda, \lambda^{\prime} ; \tau\right)$ say, between an eigenvalue $\lambda^{\prime}$ at $\tau=0$ and an eigenvalue $\lambda$ at general $\tau$, let

$$
G\left(\lambda_{1}^{\prime}, \ldots, \lambda_{N}^{\prime} \mid \lambda_{1}, \ldots, \lambda_{N} ; \tau\right)
$$

denote the Green's function for the Fokker-Planck equation (1.1) i.e. the solution which satisfies the initial condition

$$
G\left(\lambda_{1}^{\prime}, \ldots, \lambda_{N}^{\prime} \mid \lambda_{1}, \ldots, \lambda_{N} ; 0\right)=\prod_{l=1}^{N} \delta\left(\lambda_{l}-\lambda_{l}^{\prime}\right),
$$

and let $p_{0}\left(\lambda_{1}^{\prime}, \ldots, \lambda_{N}^{\prime}\right)$ denote the initial eigenvalue p.d.f., and $\rho_{(1)}(\lambda, \tau)$ denote the one-point distribution (the density) defined by (2.1). Then

$$
\begin{aligned}
S\left(\lambda, \lambda^{\prime} ; \tau\right):= & \left(\prod_{l=1}^{N} \int_{-\infty}^{\infty} d \lambda_{l}^{\prime}\right) \sum_{j=1}^{N} \delta\left(\lambda_{j}^{\prime}-\lambda^{\prime}\right) p_{0}\left(\lambda_{1}^{\prime}, \ldots, \lambda_{N}^{\prime}\right) \\
& \times\left(\prod_{l=1}^{N} \int_{-\infty}^{\infty} d \lambda_{l}\right) \sum_{j=1}^{N} \delta\left(\lambda_{j}-\lambda\right) G\left(\lambda_{1}^{\prime}, \ldots, \lambda_{N}^{\prime} \mid \lambda_{1}, \ldots, \lambda_{N} ; \tau\right)-\rho_{(1)}\left(\lambda^{\prime}, 0\right) \rho_{(1)}(\lambda, \tau) .
\end{aligned}
$$


In the thermodynamic limit, with the scaling (2.3),

$$
\rho_{(1)}(\lambda, \tau)=\rho
$$

for all $\tau$ and we expect $S$ to depend only on the difference $\lambda-\lambda^{\prime}$, so we write

$$
S\left(\lambda, \lambda^{\prime} ; \tau\right):=S\left(\lambda-\lambda^{\prime} ; \tau\right)
$$

On the basis of a non-local diffusion equation approximation to (1.1) Beenakker and Rejaei [7] have obtained a prediction for the small- $k$ behaviour of the Fourier transform

$$
\hat{S}(k ; \tau):=\int_{-\infty}^{\infty} S\left(\lambda-\lambda^{\prime} ; \tau\right) e^{i k\left(\lambda-\lambda^{\prime}\right)} d \lambda .
$$

This prediction states that

$$
\hat{S}(k ; \tau) \sim \hat{S}(k ; 0) e^{-\pi^{2} \rho^{2} \tau|k| / \gamma} \quad \text { as } \quad k \rightarrow 0 .
$$

In particular, if the initial p.d.f. is

$$
\frac{1}{C_{\beta N}} e^{-\beta W}
$$

where $W$ is given by $(1.2)$, then [1]

$$
\hat{S}(k ; 0) \sim \frac{|k|}{\pi \beta} \quad \text { as } \quad|k| \rightarrow 0,
$$

so taking the inverse transform of (2.9) gives

$$
S\left(\lambda-\lambda^{\prime} ; \tau\right) \sim \frac{1}{2 \pi^{2} \beta} \frac{\partial^{2}}{\partial \lambda^{2}} \log \left((\pi \rho \tau / \gamma)^{2}+\left(\lambda-\lambda^{\prime}\right)^{2}\right) \quad \text { for } \quad\left|\lambda-\lambda^{\prime}\right| \rightarrow \infty .
$$

When the initial eigenvalue p.d.f. is $(2.10)$ with $\beta=2$ (the Fokker-Planck equation then describes a perturbation of the unitary ensemble), the density-density correlation is well known (see e.g. [7]):

$$
S\left(\lambda-\lambda^{\prime} ; \tau\right)=\rho^{2} \int_{0}^{1} d u_{1} \cos \pi u_{1} \rho \lambda e^{\pi^{2} \rho^{2} \tau u_{1}^{2} / 2 \gamma} \int_{1}^{\infty} d u_{2} \cos \pi u_{2} \rho \lambda e^{-\pi^{2} \rho^{2} \tau u_{2}^{2} / 2 \gamma} .
$$

(with the eigenvalues $\lambda$ and $\lambda^{\prime}$ chosen in the neighbourhood of the edge of the Wigner semi-circle, the exact evaluation of $S$ is also known [8]). The asymptotic expansion of (2.12) agrees with (2.11).

In this paper we will provide the exact evaluation of $S\left(\lambda-\lambda^{\prime} ; \tau\right)$ for the initial conditions (2.2). For the initial conditions (2.2a) the above theory gives

$$
\hat{S}(k ; \tau) \sim \frac{|k|}{\pi} e^{-\pi^{2} \rho^{2}|k| \tau / \gamma} \quad \text { as } \quad|k| \rightarrow 0,
$$

while for the initial conditon $(2.2 \mathrm{~b})$

$$
\hat{S}(k ; 0) \sim 2 \frac{|k|}{4 \pi} \quad \text { as } \quad|k| \rightarrow 0,
$$

(the factor of 2 accounts for the double degeneracy in (2.2b)), and so (2.9) gives

$$
\hat{S}(k ; \tau) \sim \frac{|k|}{2 \pi} e^{-\pi^{2} \rho^{2}|k| \tau / \gamma} \quad \text { as } \quad|k| \rightarrow 0 .
$$

Now consider the correlator $R(\tau ; \alpha)$ of a linear statistic

$$
A(\tau ; \alpha):=\sum_{j=1}^{N} a\left(\lambda_{j}(\tau) / \alpha\right) .
$$


The correlator is defined as

$$
\begin{aligned}
R(\tau ; \alpha) & :=\langle(A(0 ; \alpha)-\langle A(0 ; \alpha)\rangle)(A(\tau ; \alpha)-\langle A(\tau ; \alpha)\rangle)\rangle \\
& =\int_{-\infty}^{\infty} d \lambda \int_{-\infty}^{\infty} d \lambda^{\prime} a(\lambda / \alpha) a\left(\lambda^{\prime} / \alpha\right) S\left(\lambda-\lambda^{\prime} ; \tau\right),
\end{aligned}
$$

where the averages in the first line above are over both the eigenvalues at $\tau=0$ and the eigenvalues at general $\tau$. Beenakker and Rejaei [7] used the asymptotic formula (2.9) to compute the integrated correlator

$$
\chi_{A}:=\int_{0}^{\infty} d x R\left(x^{2} ; \alpha\right)
$$

for the initial p.d.f. (2.10), in the limit $\alpha \rightarrow \infty$ (the quantity $x^{2}=\tau$ is identified in [7] as an external perturbation parameter). Thus, introducing Fourier transforms, we have

$$
\chi_{A}=\frac{\alpha}{2 \pi} \int_{0}^{\infty} d x \int_{-\infty}^{\infty} d k|\hat{a}(k)|^{2} \hat{S}\left(k / \alpha ; x^{2}\right)
$$

In the limit $\alpha \rightarrow \infty$, use of (2.9) gives, after carrying out the integration over $x$, (eq. (4.22) of [7] with $\left.\gamma \mapsto \gamma / \pi \rho_{0}\right)$

$$
\chi_{A}=\frac{1}{2 \pi^{2} \beta}\left(\frac{\gamma}{\pi \rho^{2}}\right)^{1 / 2} \int_{0}^{\infty} d k|\hat{a}(k)|^{2} k^{1 / 2} .
$$

Another consequence of (2.9) is a formula for the current-current correlation, defined formally as

$$
C\left(\lambda, \lambda^{\prime} ; \tau\right):=\left\langle\sum_{j, k=1}^{N} \frac{d \lambda_{j}(0)}{d x} \frac{d \lambda_{k}(\tau)}{d x} \delta\left(\lambda-\lambda_{j}(0)\right) \delta\left(\lambda-\lambda_{k}(\tau)\right)\right\rangle
$$

In a Brownian motion model the derivatives cannot be computed directly. However, by considering the correlator (2.14) with $A$ replaced by $d A / d x$, it can be shown that the current-current correlation is related to the density-density correlation by [7, eq. 4.14]

$$
\hat{C}\left(k ; x^{2}\right)=-\frac{1}{k^{2}} \frac{\partial^{2}}{\partial x^{2}} \hat{S}\left(k ; x^{2}\right) .
$$

Thus, from (2.9), for small- $|k|$ and the initial condition (2.10), one obtains [7]

$$
\hat{C}\left(k ; x^{2}\right) \sim \frac{2 \rho}{\beta \gamma}\left(1-2 \pi^{2} \rho^{2}|k| x^{2} / \gamma\right) e^{-\pi^{2} \rho^{2}|k| x^{2} / \gamma},
$$

or equivalently

$$
C\left(\lambda, \lambda^{\prime} ; x^{2}\right):=C\left(\lambda-\lambda^{\prime} ; x^{2}\right) \sim \frac{1}{2 \pi^{2} \beta} \frac{\partial^{2}}{\partial x^{2}} \log \left(\left(\pi \rho x^{2} / \gamma\right)^{2}+\left(\lambda-\lambda^{\prime}\right)^{2}\right)
$$

for large- $\left|\lambda-\lambda^{\prime}\right|$.

\subsection{The distribution of the initial rate of change of the eigenvalues}

As commented in the above section, the parameter $x:=\tau^{1 / 2}$ can be identified with an external perturbing

parameter. For the initial conditions (2.2b) a quantity of interest [9] associated with this interpretation is the distribution of the scaled repulsion between degenerate levels as the perturbation is initialized:

$$
A(\alpha):=\lim _{\tau \rightarrow 0} \lim _{N \rightarrow \infty}\left\langle\frac{2}{N} \sum_{j=1}^{N / 2} \delta\left(\alpha-\frac{\lambda_{2 j}(\tau)-\lambda_{2 j-1}(\tau)}{x}\right)\right\rangle
$$




$$
\begin{aligned}
& =\lim _{\tau \rightarrow 0} \lim _{N \rightarrow \infty}\left\langle\frac{1}{2 \pi} \frac{2}{N} \sum_{j=1}^{N / 2} \int_{-\infty}^{\infty} d p e^{i p\left(\alpha-\left(\lambda_{2 j}(\tau)-\lambda_{2 j-1}(\tau)\right) / \tau^{1 / 2}\right)}\right\rangle \\
& =\lim _{\tau \rightarrow 0} \frac{1}{2 \pi \rho} \int_{-\infty}^{\infty} d p e^{i p \alpha} \int_{-\infty}^{\infty} d \lambda \rho_{(2)}^{T}(\lambda ; \tau) e^{-i p \lambda / \tau^{1 / 2}} \\
& =\lim _{\tau \rightarrow 0} \frac{\tau^{1 / 2}}{\rho} \rho_{(2)}^{T}\left(\alpha \tau^{1 / 2} ; \tau\right)
\end{aligned}
$$

The exact result (2.4) gives [9]

$$
A(\alpha)=\left(\frac{\gamma}{2 \pi}\right)^{1 / 2} \gamma \alpha^{2} e^{-\gamma \alpha^{2} / 2}
$$

A quantity of interest for general initial conditions is the distribution of the initial rate of change of the eigenvalues with respect to $x$ :

$$
\begin{aligned}
f(v) & :=\lim _{\tau \rightarrow 0}\left\langle\frac{1}{N} \sum_{j=1}^{N} \delta\left(v-\frac{\lambda_{j}(\tau)-\lambda_{j}(0)}{x}\right)\right\rangle \\
& =\lim _{\tau \rightarrow 0} \frac{1}{2 \pi \rho} \int_{-\infty}^{\infty} d p e^{i p v} \int_{-\infty}^{\infty} d \lambda S(\lambda, \tau) e^{-i \lambda p / \tau^{1 / 2}} \\
& =\lim _{\tau \rightarrow 0} \frac{\tau^{1 / 2}}{\rho} S\left(v \tau^{1 / 2} ; \tau\right)
\end{aligned}
$$

Using the exact result (2.4) for initial conditions with unitary symmetry, (2.19) gives [10] the Gaussian distribution

$$
f(v)=\left(\frac{\gamma}{2 \pi}\right)^{1 / 2} e^{-v^{2} \gamma / 2}
$$

\section{Exact solution for initial conditions with orthogonal symme- try}

\subsection{Green's function for $\beta=2$ in periodic model}

Our interest is in calculating the correlation (2.8) in the thermodynamic limit. For this purpose we can replace the potential $W$ in (1.2) by its periodic version

$$
W=-\sum_{1 \leq j<k \leq N} \log \left|\sin \pi\left(\lambda_{k}-\lambda_{j}\right) / L\right|, \quad-L / 2 \leq \lambda_{j} \leq L / 2
$$

This gives the Fokker-Planck equation of the Dyson Brownian motion model for the circular ensemble of unitary random matrices [11].

In general the equilibrium solution of (1.1) is

$$
p=\frac{1}{C_{N \beta}} e^{-\beta W},
$$

and the operator

$$
e^{\beta W / 2} \mathcal{L} e^{-\beta W / 2}
$$

is Hermitian [12]. With $\beta=2$ and $W$ given by (3.1), a standard calculation gives

$$
-e^{\beta W / 2} \mathcal{L} e^{-\beta W / 2}=-\frac{1}{2} \sum_{j=1}^{N} \frac{\partial^{2}}{\partial \lambda_{j}^{2}}-E_{0}
$$


where

$$
E_{0}=\left(\frac{2 \pi}{L}\right)^{2} \frac{N\left(N^{2}-1\right)}{12}
$$

The well known reason for the tractibility of the case $\beta=2$ is thus revealed: the Hermitian operator on the r.h.s. of (3.3) is the Schrödinger operator for non-interacting particles. To reclaim the correct equilibrium solution (3.2) the particles are assumed to be impenetrable, so that the wavefunction vanishes if two particles are at the same point.

Thus if $g\left(\lambda_{1}^{(0)}, \ldots, \lambda_{N}^{(0)} \mid \lambda_{1}, \ldots, \lambda_{N} ; \tau\right)$ denotes the Green's function satisfying the imaginary time Schrödinger equation

$$
-\frac{\partial g}{\partial \tau}=-\frac{1}{2} \sum_{j=1}^{N} \frac{\partial^{2}}{\partial \lambda_{j}^{2}}-E_{0},
$$

then the Green's function $G$ for the Fokker-Planck equation (1.1), with $\beta=2$ and $W$ given by (3.1), is related to $g$ by

$$
G\left(\lambda_{1}^{(0)}, \ldots, \lambda_{N}^{(0)} \mid \lambda_{1}, \ldots, \lambda_{N} ; \tau\right)=e^{-\left(W\left(\lambda_{1}, \ldots, \lambda_{N}\right)-W\left(\lambda_{1}^{(0)}, \ldots, \lambda_{N}^{(0)}\right)\right)} g\left(\lambda_{1}^{(0)}, \ldots, \lambda_{N}^{(0)} \mid \lambda_{1}, \ldots, \lambda_{N} ; \tau\right)
$$

Due to the impenetrability condition of the last paragraph we can suppose that the eigenvalues are ordered

$$
-L / 2 \leq \lambda_{1}<\ldots<\lambda_{N} \leq L / 2,
$$

(and similarly the initial eigenvalues $\lambda_{j}^{(0)}$ ), which allows us to write

$$
e^{-\left(W\left(\lambda_{1}, \ldots, \lambda_{N}\right)-W\left(\lambda_{1}^{(0)}, \ldots, \lambda_{N}^{(0)}\right)\right)}=\prod_{1 \leq j<k \leq N} \frac{\sin \pi\left(\lambda_{k}-\lambda_{j}\right) / L}{\sin \pi\left(\lambda_{k}^{(0)}-\lambda_{j}^{(0)}\right) / L} .
$$

For $N$ even (3.7) is anti-periodic under the mapping $\lambda_{k} \mapsto \lambda_{k}+L$. We then require that $g$ be antiperiodic under the same mapping, so by (3.5), $G$ will be periodic.

It is straightforward to check $[6,13]$ that for $N$ even the Green's function solution of $(3.4)$ satisfying the required conditions is

$$
g\left(\lambda_{1}^{(0)}, \ldots, \lambda_{N}^{(0)} \mid \lambda_{1}, \ldots, \lambda_{N} ; \tau\right)=e^{\tau E_{0} / \gamma} \operatorname{det}\left[\frac{1}{L} \theta_{2}\left(\pi\left(\lambda_{j}-\lambda_{k}^{(0)}\right) / L ; q\right)\right]_{j, k=1, \ldots, N}
$$

where

$$
q:=e^{-2 \pi^{2} \tau / \gamma L^{2}} \quad \theta_{2}(z ; q):=\sum_{n=-\infty}^{\infty} q^{(n-1 / 2)^{2}} e^{2 i z(n-1 / 2)},
$$

(for $N$ odd $\theta_{2}$ is to be replaced by $\theta_{3}$ ). Thus we have that for $N$ even the required Green's function is given by substituting (3.7) and (3.8) in (3.5).

\subsection{The integration technique}

We want to calculate the density-density correlation (2.8) with $G$ given as above and the initial condition

$$
p\left(\lambda_{1}^{(0)}, \ldots, \lambda_{N}^{(0)}\right)=\frac{1}{C_{1 N}} \prod_{1 \leq j<k \leq N}\left|\sin \pi\left(\lambda_{k}^{(0)}-\lambda_{j}^{(0)}\right) / L\right|
$$

Since we are considering the periodic model the integrations in (2.8) must now be from $-L / 2$ to $L / 2$ and

$$
\rho_{(1)}\left(\lambda^{\prime} ; 0\right)=\rho_{(1)}(\lambda ; \tau)=N / L=\rho .
$$


The exact value of the normalization $C_{1 N}$ is known but will not be needed in our subsequent analysis.

To calculate (2.8) we will consider the generalized partition function

$$
Z(a, b):=\prod_{l=1}^{N}\left(\int_{-L / 2}^{L / 2} d \lambda_{l}^{(0)} b\left(\lambda_{l}^{(0)}\right) \int_{-L / 2}^{L / 2} d \lambda_{l} a\left(\lambda_{l}\right)\right) p\left(\lambda_{1}^{(0)}, \ldots, \lambda_{N}^{(0)}\right) G\left(\lambda_{1}^{(0)}, \ldots, \lambda_{N}^{(0)} \mid \lambda_{1}, \ldots, \lambda_{N} ; \tau\right)
$$

and use the formula

$$
S\left(\lambda, \lambda^{\prime} ; \tau\right)=\left.\frac{\delta^{2}}{\delta a(\lambda) \delta b\left(\lambda^{\prime}\right)} \log Z(a, b)\right|_{a=b=1} .
$$

Substituting for $p$ and $G$ we have

$$
\begin{aligned}
Z(a, b) & =C \prod_{l=1}^{N}\left(\int_{-L / 2}^{L / 2} d \lambda_{l}^{(0)} b\left(\lambda_{l}^{(0)}\right) \int_{-L / 2}^{L / 2} d \lambda_{l} a\left(\lambda_{l}\right)\right) \prod_{1 \leq j<k \leq N} \operatorname{sgn}\left(\lambda_{k}^{(0)}-\lambda_{j}^{(0)}\right) \\
& \times \operatorname{det}\left[\frac{1}{L} \theta_{2}\left(\pi\left(\lambda_{j}-\lambda_{k}^{(0)}\right) / L ; q\right)\right]_{j, k=1, \ldots, N} \operatorname{det}\left[e^{2 \pi i \lambda_{j}(k-(N+1) / 2) / L}\right]_{j, k=1, \ldots, N},
\end{aligned}
$$

where $C$ is independent of $\lambda_{l}^{(0)}, \lambda_{l}$, and the Vandermonde determinant formula

$$
\prod_{1 \leq j<k \leq N} 2 \sin \pi\left(\lambda_{k}-\lambda_{j}\right) / L=i^{-N(N-1) / 2} \operatorname{det}\left[e^{2 \pi i \lambda_{j}(k-(N+1) / 2) / L}\right]_{j, k=1, \ldots, N}
$$

has been used.

To simplify (3.13), we note that since

$$
\operatorname{det}\left[\frac{1}{L} \theta_{2}\left(\pi\left(\lambda_{j}-\lambda_{k}^{(0)}\right) / L ; q\right)\right]_{j, k=1, \ldots, N}
$$

is anti-symmetric in each $\lambda_{l}$, we can make the replacement

$$
\operatorname{det}\left[e^{2 \pi i \lambda_{j}(k-(N+1) / 2) / L}\right]_{j, k=1, \ldots, N} \mapsto N ! \prod_{j=1}^{N} e^{2 \pi i \lambda_{j}(j-(N+1) / 2) / L}
$$

in the integrand. The integrations over the $\lambda_{l}$ can now be performed row-by-row to give

$$
Z(a, b)=C N ! \prod_{l=1}^{N}\left(\int_{-L / 2}^{L / 2} d \lambda_{l}^{(0)} b\left(\lambda_{l}^{(0)}\right)\right) \prod_{1 \leq j<k \leq N} \operatorname{sgn}\left(\lambda_{k}^{(0)}-\lambda_{j}^{(0)}\right) \operatorname{det}\left[A_{j}\left(\lambda_{k}^{(0)}\right)\right]_{j, k=1, \ldots, N}
$$

where

$$
A_{j}\left(\lambda_{k}^{(0)}\right):=\frac{1}{L} \int_{-L / 2}^{L / 2} d \lambda a(\lambda) \theta_{2}\left(\pi\left(\lambda-\lambda_{k}^{(0)}\right) / L ; q\right) e^{2 \pi i \lambda(j-(N+1) / 2) / L} .
$$

The integrations over the $\lambda_{l}^{(0)}$ can now be done using the method of integration over alternate variables [1] to give

$$
Z(a, b)=C N !^{2} \operatorname{det}\left[H_{j k}\right]_{j, k=1, \ldots, N}^{1 / 2}
$$

where

$$
H_{j k}:=\int_{-L / 2}^{L / 2} d \lambda^{(0)} b\left(\lambda^{(0)}\right) \int_{-L / 2}^{L / 2} d \lambda_{1}^{(0)} b\left(\lambda_{1}^{(0)}\right) A_{j}\left(\lambda^{(0)}\right) A_{k}\left(\lambda_{1}^{(0)}\right) \operatorname{sgn}\left(\lambda^{(0)}-\lambda_{1}^{(0)}\right)
$$

Since

$$
\rho=\left.\frac{1}{2 \operatorname{det}\left[H_{j k}\right]} \frac{\delta}{\delta a(\lambda)} \operatorname{det}\left[H_{j k}\right]\right|_{a=b=1}=\left.\frac{1}{2 \operatorname{det}\left[H_{j k}\right]} \frac{\delta}{\delta b\left(\lambda^{\prime}\right)} \operatorname{det}\left[H_{j k}\right]\right|_{a=b=1}
$$

(3.12) and (3.17) give

$$
S\left(\lambda, \lambda^{\prime} ; \tau\right)=-2 \rho^{2}+\left.\frac{1}{2 \operatorname{det}\left[H_{j k}\right]} \frac{\delta^{2}}{\delta a(\lambda) \delta b\left(\lambda^{\prime}\right)} \operatorname{det}\left[H_{j k}\right]\right|_{a=b=1} .
$$




\subsection{Calculation of the determinants}

From (3.16), and the definition of $\theta_{2}$, when $a=b=1$

$$
A_{j}(\lambda)=e^{2 \pi i(j-(N+1) / 2) \lambda / L} q^{(j-(N+1) / 2)^{2}} .
$$

With this value of $A_{j}(\lambda)$ we have

$$
\int_{-L / 2}^{L / 2} d \lambda A_{j}(\lambda) \operatorname{sgn}\left(\lambda-\lambda^{\prime}\right)=-\frac{L}{\pi i(j-(N+1) / 2)} A_{j}\left(\lambda^{\prime}\right)
$$

Substituting (3.21) and (3.22) in (3.18) shows that when $a=b=1$

$$
H_{j k}=h_{j} \delta_{N+1-j-k, 0},
$$

where

$$
h_{j}:=-\frac{L^{2}}{\pi i(j-(N+1) / 2)} q^{2(j-(N+1) / 2)^{2}}
$$

Hence $\left[H_{j k}\right]_{j, k=1, \ldots, N}$ is only non-zero along the right-to-left diagonal.

Taking the functional derivative $\delta / \delta b\left(\lambda^{\prime}\right)$ in row $j$ and setting $a=b=1$ gives

$$
\begin{aligned}
I_{j, k}\left(\lambda^{\prime}\right) & :=\int_{-L / 2}^{L / 2} d \lambda\left(-A_{j}(\lambda) A_{k}\left(\lambda^{\prime}\right)+A_{j}\left(\lambda^{\prime}\right) A_{k}(\lambda)\right) \operatorname{sgn}\left(\lambda^{\prime}-\lambda\right) \\
& =e^{2 \pi i(j+k-(N+1)) \lambda^{\prime} / L} q^{(j-(N+1) / 2)^{2}+(k-(N+1) / 2)^{2}} \frac{L}{\pi i}\left(\frac{1}{k-(N+1) / 2}-\frac{1}{j-(N+1) / 2}\right) .
\end{aligned}
$$

Taking the functional derivative $\delta / \delta a(\lambda)$ in row $j^{\prime}$ and setting $a=b=1$ gives, for $j^{\prime} \neq j$

$$
\begin{aligned}
J_{j^{\prime}, k}(\lambda):= & \frac{1}{L} \int_{-L / 2}^{L / 2} d \lambda^{(0)} \int_{-L / 2}^{L / 2} d \lambda_{1}^{(0)}\left(\theta_{2}\left(\pi\left(\lambda-\lambda^{(0)}\right) / L ; q\right) e^{2 \pi i\left(j^{\prime}-(N+1) / 2\right) \lambda / L} A_{k}\left(\lambda_{1}^{(0)}\right)\right. \\
& \left.+\theta_{2}\left(\pi\left(\lambda-\lambda_{1}^{(0)}\right) / L ; q\right) e^{2 \pi i(k-(N+1) / 2) \lambda / L} A_{j}\left(\lambda^{(0)}\right)\right) \operatorname{sgn}\left(\lambda^{(0)}-\lambda_{1}^{(0)}\right) \\
= & q^{2(k-(N+1) / 2)^{2}} e^{2 \pi i\left(j^{\prime}+k-(N+1)\right) \lambda / L}\left(\frac{L}{\pi i(k-(N+1) / 2)}\right)-\left(j^{\prime} \leftrightarrow k\right),
\end{aligned}
$$

while for $j^{\prime}=j$ we obtain

$$
\begin{aligned}
K_{j, k}( & \left.\lambda, \lambda^{\prime}\right) \\
:= & \frac{1}{L} \theta_{2}\left(\pi\left(\lambda-\lambda^{\prime}\right) / L ; q\right) \int_{-L / 2}^{L / 2} d \lambda^{(0)}\left(e^{2 \pi i(j-(N+1) / 2) \lambda / L} A_{k}\left(\lambda^{(0)}\right)-(j \leftrightarrow k)\right) \operatorname{sgn}\left(\lambda^{\prime}-\lambda^{(0)}\right) \\
& +\left(e^{2 \pi i(j-(N+1) / 2) \lambda / L} A_{k}\left(\lambda^{\prime}\right)-(j \leftrightarrow k)\right) \frac{1}{L} \int_{-L / 2}^{L / 2} d \lambda^{(0)} \operatorname{sgn}\left(\lambda^{(0)}-\lambda^{\prime}\right) \theta_{2}\left(\pi\left(\lambda-\lambda^{(0)}\right) / L ; q\right) \\
= & \frac{1}{L} \theta_{2}\left(\pi\left(\lambda-\lambda^{\prime}\right) / L ; q\right)\left(\frac{L}{\pi i(k-(N+1) / 2)} q^{(k-(N+1) / 2)^{2}} e^{2 \pi i\left(j \lambda+k \lambda^{\prime}-\left(\lambda+\lambda^{\prime}\right)(N+1) / 2\right) / L}-(j \leftrightarrow k)\right) \\
& +\left(q^{(k-(N+1) / 2)^{2}} e^{2 \pi i\left(j \lambda+k \lambda^{\prime}-\left(\lambda+\lambda^{\prime}\right)(N+1) / 2\right) / L}-(j \leftrightarrow k)\right) \frac{1}{L} \int_{-L / 2}^{L / 2} d \lambda^{(0)} \operatorname{sgn}\left(\lambda^{(0)}-\lambda^{\prime}\right) \theta_{2}\left(\pi\left(\lambda-\lambda^{(0)}\right) / L ; q\right)
\end{aligned}
$$

From (3.23), with $a=b=1$, in each row $j^{\prime \prime}\left(\neq j, j^{\prime}\right)$ the only non-zero term is along the right to left diagonal and is given by $h_{j^{\prime \prime}}$. Expanding the determinant along this diagonal shows that (3.20) reduces to

$$
S\left(\lambda^{\prime}, \lambda ; \tau\right)=-2 \rho^{2}+\frac{1}{2}\left(\sum_{\substack{j, j^{\prime}=1 \\ j \neq j^{\prime}}}^{N}\left(\frac{I_{j, N+1-j}\left(\lambda^{\prime}\right) J_{j^{\prime}, N+1-j^{\prime}}(\lambda)}{h_{j} h_{j^{\prime}}}-\left(j \leftrightarrow j^{\prime}, N+1-j\left(j^{\prime}\right) \text { fixed }\right)\right)\right.
$$




$$
\left.+\sum_{j=1}^{N} \frac{K_{j, N+1-j}\left(\lambda, \lambda^{\prime}\right)}{h_{j}}\right) .
$$

We note that the above expression is unchanged if we remove the restriction $j \neq j^{\prime}$ in the double sum. Doing this, and noting

$$
\sum_{j=1}^{N} \frac{I_{j, N+1-j}\left(\lambda^{\prime}\right)}{h_{j}}=\sum_{j^{\prime}=1}^{N} \frac{J_{j^{\prime}, N+1-j^{\prime}}(\lambda)}{h_{j}^{\prime}}=2 \rho,
$$

(3.27) reads

$$
S\left(\lambda^{\prime}, \lambda ; \tau\right)=\frac{1}{2}\left(\sum_{j=1}^{N} \frac{K_{j, N+1-j}\left(\lambda, \lambda^{\prime}\right)}{h_{j}}-\sum_{j, j^{\prime}=1}^{N} \frac{I_{j, N+1-j^{\prime}}\left(\lambda^{\prime}\right) J_{j^{\prime}, N+1-j}(\lambda)}{h_{j} h_{j^{\prime}}}\right) .
$$

The quantities in the above expressions are given explicitly by (3.23b) and (3.24)-(3.26). We see immediately that the double sum is a Riemann approximation to a definite integral, with the approximation becoming exact in the thermodynamic limit. Taking the thermodynamic limit of the first term requires an intermediate step: from (3.26) the limiting behaviour of $\theta_{2}$ is required.

From the Poisson summation formula

$$
\sum_{n=-\infty}^{\infty} e^{-\epsilon(n-1 / 2)^{2}} e^{2 i(n-1 / 2) z}=\left(\frac{\pi}{\epsilon}\right)^{1 / 2} \sum_{m=-\infty}^{\infty}(-1)^{m} e^{-(z+\pi m)^{2} / \epsilon} .
$$

Hence with $z=\pi\left(\lambda-\lambda^{\prime}\right) / L$ and $\epsilon=2 \pi^{2} \tau / \gamma L^{2}$

$$
\begin{aligned}
\frac{1}{L} \theta_{2}\left(\pi\left(\lambda-\lambda^{\prime}\right) / L ; q\right) & =\frac{1}{L}\left(\frac{\gamma L^{2}}{2 \pi \tau}\right)^{1 / 2} \sum_{m=-\infty}^{\infty}(-1)^{m} e^{-\gamma\left(\lambda-\lambda^{\prime}+m L\right)^{2} / 2 \tau} \\
& \sim\left(\frac{\gamma}{2 \pi \tau}\right)^{1 / 2} e^{-\gamma\left(\lambda-\lambda^{\prime}\right)^{2} / 2 \tau} \quad \text { as } \quad L \rightarrow \infty \\
& =\rho \int_{-\infty}^{\infty} d u_{1} e^{-2 \tau\left(\pi \rho u_{1}\right)^{2} / \gamma} \cos 2 \pi u_{1} \rho\left(\lambda-\lambda^{\prime}\right) .
\end{aligned}
$$

Using this asymptotic formula, and from the remarks in the paragraph above, we thus have that in the thermodyamic limit

$$
\begin{aligned}
& S\left(\lambda^{\prime}, \lambda ; \tau\right)= \\
& \quad \rho^{2} \int_{-\infty}^{\infty} d u_{1} e^{-2 \tau\left(\pi \rho u_{1}\right)^{2} / \gamma} \cos 2 \pi u_{1} \rho\left(\lambda-\lambda^{\prime}\right) \int_{-1 / 2}^{1 / 2} d u_{2} e^{2 \tau\left(\pi \rho u_{1}\right)^{2} / \gamma} \cos 2 \pi u_{2} \rho\left(\lambda-\lambda^{\prime}\right) \\
& \quad+\rho^{2} \int_{-\infty}^{\infty} d u_{1} \frac{e^{-2 \tau\left(\pi \rho u_{1}\right)^{2} / \gamma}}{u_{1}} \sin 2 \pi u_{1} \rho\left(\lambda-\lambda^{\prime}\right) \int_{-1 / 2}^{1 / 2} d u_{2} u_{2} e^{2 \tau\left(\pi \rho u_{1}\right)^{2} / \gamma} \sin 2 \pi u_{2} \rho\left(\lambda-\lambda^{\prime}\right) \\
& \quad-\frac{\rho^{2}}{2} \int_{-1 / 2}^{1 / 2} d u_{1} \int_{-1 / 2}^{1 / 2} d u_{2}\left(u_{1}+u_{2}\right)\left(\frac{1}{u_{1}} e^{-2 \tau(\pi \rho)^{2}\left(u_{1}^{2}-u_{2}^{2}\right) / \gamma}+\frac{1}{u_{2}} e^{-2 \tau(\pi \rho)^{2}\left(u_{2}^{2}-u_{1}^{2}\right) / \gamma}\right) e^{2 \pi i\left(u_{1}-u_{2}\right) \rho\left(\lambda^{\prime}-\lambda\right)}
\end{aligned}
$$

where to obtain the second term the formula

$$
\begin{gathered}
\int_{-\infty}^{\infty} d \lambda^{(0)} \operatorname{sgn}\left(\lambda^{(0)}-\lambda^{\prime}\right) \int_{-\infty}^{\infty} d u_{1} e^{-2 \tau\left(\pi \rho u_{1}\right)^{2} / \gamma} \cos 2 \pi u_{1} \rho\left(\lambda^{(0)}-\lambda\right) \\
=-\frac{1}{\pi} \int_{-\infty}^{\infty} d u_{1} \frac{e^{-2 \tau\left(\pi \rho u_{1}\right)^{2} / \gamma}}{u_{1}} \sin 2 \pi u_{1} \rho\left(\lambda-\lambda^{\prime}\right)
\end{gathered}
$$

has been used. 
Expressing the double integrals as single integrals and simplifying gives

$$
\begin{aligned}
& S\left(\lambda^{\prime}, \lambda ; \tau\right):=S\left(\lambda^{\prime}-\lambda ; \tau\right) \\
& \quad=\rho^{2}\left(\int_{1}^{\infty} d u_{1} e^{-\tau\left(\pi \rho u_{1}\right)^{2} / 2 \gamma} \cos \pi u_{1} \rho\left(\lambda-\lambda^{\prime}\right) \int_{0}^{1} d u_{2} e^{\tau\left(\pi \rho u_{2}\right)^{2} / 2 \gamma} \cos \pi u_{2} \rho\left(\lambda-\lambda^{\prime}\right)\right. \\
& \quad+\int_{1}^{\infty} d u_{1} \frac{e^{-\tau\left(\pi \rho u_{1}\right)^{2} / 2 \gamma}}{u_{1}} \sin \pi u_{1} \rho\left(\lambda-\lambda^{\prime}\right) \int_{0}^{1} d u_{2} u_{2} e^{\tau\left(\pi \rho u_{2}\right)^{2} / 2 \gamma} \sin \pi u_{2} \rho\left(\lambda-\lambda^{\prime}\right)
\end{aligned}
$$

We note that the first term is precisely the density-density correlation for initial conditions with unitary symmetry given by (2.11), while the second term is precisely that which occurs in the two-point distribution function (2.4) (after replacing $\tau$ by $2 \tau$ ) with $\beta_{0}=1$ (after replacing $\tau$ by $2 \tau$ ). The large $\tau$, $\lambda-\lambda^{\prime}$ expansion of the above integrals is obtained by expanding the integrands in the neighbourhood of $u_{1}, u_{2}=1$. The behaviour (2.1) with $\beta=1$ is verified.

The Fourier transform with respect to $\lambda-\lambda^{\prime}$ of each of the products in (3.31) have been calculated in ref. [7] and [5] respectively. Thus we have

$$
\hat{S}(k ; \tau)=\hat{S}_{1}(k ; \tau)+\hat{S}_{2}(k ; \tau)
$$

where

$$
\hat{S}_{1}(k ; \tau):=\frac{\gamma}{\tau \pi|k|} \exp \left(-\tau \pi|k| q_{\max } \rho / \gamma\right) \sinh \left(\tau \pi|k| q_{\min } \rho / \gamma\right)
$$

with

$$
q_{\min }:=\min (1,|k| / 2 \pi \rho) \quad q_{\max }:=\max (1,|k| / 2 \pi \rho),
$$

and

$$
\begin{gathered}
\hat{S}_{2}(k ; \tau):=\frac{\gamma}{2 \pi \tau|k|}\left(1-e^{-\tau k^{2} / \gamma}\right)-\frac{|k|}{2 \pi} e^{\tau k^{2} / 2 \gamma} \int_{1}^{1+|k| / \pi \rho} d k_{1} \frac{1}{k_{1}} e^{-\pi \tau \rho|k| k_{1} / \gamma}, \quad 0 \leq|k| \leq 2 \pi \rho, \quad \\
\hat{S}_{2}(k ; \tau):=\frac{\gamma}{\pi \tau|k|} e^{-\tau k^{2} / 2 \gamma} \sinh \pi \tau \rho|k| / \gamma-\frac{|k|}{2 \pi} e^{\tau k^{2} / 2 \gamma} \int_{-1+|k| / \pi \rho}^{1+|k| / \pi \rho} d k_{1} \frac{1}{k_{1}} e^{-\pi \tau \rho|k| k_{1} / \gamma}, \quad|k| \geq 2 \pi \rho .
\end{gathered}
$$

Although the small- $|k|$ behaviour of this expression agrees with (2.13a), we see that there is also a singularity at $|k|=2 \pi \rho$. This corresponds to oscillatory terms in the large- $\left|\lambda-\lambda^{\prime}\right|$ expansion of (3.31) and indicates a preference for crystalline ordering with spacing $1 / 2 \pi \rho$.

For $\tau,\left|\lambda-\lambda^{\prime}\right| \rightarrow 0$ (3.31) gives

$$
S\left(\lambda^{\prime}-\lambda ; \tau\right) \sim \frac{\rho}{\tau^{1 / 2}} \int_{0}^{\infty} d u_{1} e^{-u_{1}^{2} / 2 \gamma} \cos \pi u_{1}\left(\lambda-\lambda^{\prime}\right) / \tau^{1 / 2}
$$

Thus, from (2.17),

$$
\begin{aligned}
f(v) & =\int_{0}^{\infty} d u_{1} e^{-u_{1}^{2} / 2 \gamma} \cos \pi u_{1} v \\
& =\left(\frac{\gamma}{2 \pi}\right)^{1 / 2} e^{-v^{2} \gamma / 2},
\end{aligned}
$$

which is identical to the result (2.20) for initial conditions with unitary symmetry. It is also identical to the result found by Simons and Altshuler [10] when the initial and final distributions are given by (2.2a) (i.e. for a perturbation of the eigenvalue p.d.f. with orthogonal symmetry). 


\section{Exact solution with symplectic symmetry initial conditions}

The Green's function for the Dyson Brownian motion model for the circular ensemble with $\beta=2$ and $N$ even is given by substituting (3.7) and (3.8) in (3.5), and is of course valid independent of the particular initial conditions. In this section we want to calculate the density-density correlation with the initial conditions $(2.2 \mathrm{~b})$.

The first step is to take the limits

$$
\lambda_{2 j-1}^{(0)} \mapsto \lambda_{2 j}^{(0)} \quad j=1, \ldots, N / 2
$$

in the Green's function. We readily find, assuming the ordering (3.6)

$$
\begin{aligned}
G= & \left(\frac{L}{\pi}\right)^{N} e^{\tau E_{0} / \gamma} \frac{\prod_{1 \leq j<k \leq N} \sin \pi\left(\lambda_{k}-\lambda_{j}\right) / L}{\prod_{1 \leq j<k \leq N / 2} \sin ^{4} \pi\left(\lambda_{2 k}^{(0)}-\lambda_{2 j}^{(0)}\right) / L} \\
& \times \operatorname{det}\left[\frac{1}{L} \theta_{2}\left(\pi\left(\lambda_{j}-\lambda_{2 k}^{(0)}\right) / L ; q\right) \quad \frac{\partial}{\partial \lambda_{2 k}^{(0)}} \frac{1}{L} \theta_{2}\left(\pi\left(\lambda_{j}-\lambda_{2 k}^{(0)}\right) / L ; q\right)\right]_{\substack{j=1, \ldots, N \\
k=1, \ldots, N / 2}}^{\substack{c \\
j}}
\end{aligned}
$$

To obtain an explicit expression for the generalized partition function (3.11) we multiply $G$ by

$$
p\left(\lambda_{2}^{(0)}, \ldots, \lambda_{N}^{(0)}\right)=\frac{1}{C_{4(N / 2)}} \prod_{1 \leq j<k \leq N / 2} \sin ^{4} \pi\left(\lambda_{2 k}^{(0)}-\lambda_{2 j}^{(0)}\right) / L .
$$

This gives

$$
\begin{aligned}
& Z(a, b):=\prod_{l=1}^{N}\left(\int_{-L / 2}^{L / 2} d \lambda_{l}^{(0)} b\left(\lambda^{(0)}\right) \int_{-L / 2}^{L / 2} d \lambda_{l} a\left(\lambda_{l}\right)\right) \\
& \quad \times \operatorname{det}\left[\frac{1}{L} \theta_{2}\left(\pi\left(\lambda_{j}-\lambda_{2 k}^{(0)}\right) / L ; q\right) \quad \frac{\partial}{\partial \lambda_{2 k}^{(0)}} \frac{1}{L} \theta_{2}\left(\pi\left(\lambda_{j}-\lambda_{2 k}^{(0)}\right) / L ; q\right)\right]_{\substack{j=1, \ldots, N \\
k=1, \ldots, N / 2}} \operatorname{det}\left[e^{\left.2 \pi i \lambda_{j}(k-(N+1) / 2) / L\right)}\right]_{j, k=1, \ldots, N}
\end{aligned}
$$

where we have used the Vandermonde determinant formula (3.14).

The integrations over $\lambda_{1}, \ldots, \lambda_{N}$ can be performed row-by-row while the integrations over $\lambda_{2}^{(0)}, \ldots, \lambda_{N}^{(0)}$ yield a pfaffian structure [1]. We obtain

$$
Z(a, b)=C\left(\operatorname{det}\left[\int_{-L / 2}^{L / 2} d \lambda^{(0)} b\left(\lambda^{(0)}\right) U_{j k}\left(\lambda^{(0)}\right)\right]_{j, k=1, \ldots, N}\right)^{1 / 2}
$$

where

$$
U_{j k}\left(\lambda^{(0)}\right):=A_{j}\left(\lambda^{(0)}\right) \frac{\partial}{\partial \lambda^{(0)}} A_{k}\left(\lambda^{(0)}\right)-(j \leftrightarrow k)
$$

and $A_{j}$ is given by (3.16). The density-density correlation can be calculated from (4.3) using (3.20) with $H_{j k}$ replaced by $U_{j k}$ and $-2 \rho^{2}$ replaced by $-\rho^{2}$. In fact the calculation is very similar to that presented in Section 3.3.

Thus with $a=b=1$

$$
U_{j k}=u_{j} \delta_{N+1-j-k, 0}
$$

where

$$
u_{j}:=2 \pi i(k-j) q^{2(j-(N+1) / 2)^{2}}
$$


so that $\left[U_{j k}\right]_{j, k=1, \ldots, N}$ is non-zero only along the right-to-left diagonal (c.f. (3.23)). Taking the functional derivative $\delta / \delta a(\lambda)$ in row $j$ and setting $a=b=1$ gives

$$
\left.V_{j k}(\lambda):=\frac{4 \pi i}{L} e^{2 \pi i \lambda(j+k-(N+1)) / L}((k-(N+1) / 2)) q^{2(k-(N+1) / 2)^{2}}-(j \leftrightarrow k)\right)
$$

while taking the functional derivative $\delta / \delta b\left(\lambda^{\prime}\right)$ in row $j^{\prime}$ gives

$$
W_{j^{\prime} k}\left(\lambda^{\prime}\right)=\frac{2 \pi i}{L}\left(k-j^{\prime}\right) q^{(k-(N+1) / 2)^{2}+\left(j^{\prime}-(N+1) / 2\right)^{2}} e^{2 \pi i \lambda^{\prime}\left(j^{\prime}+k-(N+1)\right) / L}
$$

for $j \neq j^{\prime}$, and

$$
\begin{aligned}
X_{j k}\left(\lambda, \lambda^{\prime}\right) \\
=\frac{1}{L} e^{2 \pi i \lambda(j-(N+1) / 2) / L} \theta_{2}\left(\pi\left(\lambda-\lambda^{\prime}\right) / L ; q\right) \frac{\partial}{\partial \lambda^{\prime}} A_{k}\left(\lambda^{\prime}\right)-(j \leftrightarrow k) \\
\quad+\frac{1}{L} e^{2 \pi i \lambda(k-(N+1) / 2) / L} e^{2 \pi i \lambda^{\prime}(j-(N+1) / 2) / L} q^{(j-(N+1) / 2)^{2}} \frac{\partial}{\partial \lambda^{\prime}} \theta_{2}\left(\pi\left(\lambda-\lambda^{\prime}\right) / L ; q\right)-(j \leftrightarrow k)
\end{aligned}
$$

for $j=j^{\prime}$.

Since in each row $j^{\prime \prime}\left(\neq j, j^{\prime}\right)$ the only non-zero element in the denominator of (3.20) (with $H_{j k}$ replaced by $U_{j k}$ ) is along the right-left diagonal and is given by $u_{j^{\prime}}$, we conclude (c.f (3.28))

$$
S\left(\lambda^{\prime}, \lambda ; \tau\right)=\frac{1}{2}\left(\sum_{j=1}^{N} \frac{X_{j, N+1-j}\left(\lambda, \lambda^{\prime}\right)}{u_{j}}-\sum_{j, j^{\prime}=1}^{N} \frac{V_{j, N+1-j^{\prime}}(\lambda) W_{j^{\prime}, N+1-j}\left(\lambda^{\prime}\right)}{u_{j} u_{j^{\prime}}}-\left(j \leftrightarrow j^{\prime}\right)\right)
$$

where we have used the fact that

$$
\sum_{j=1}^{N} \frac{V_{j, N+1-j}(\lambda)}{u_{j}}=2 \sum_{j^{\prime}=1}^{N} \frac{W_{j^{\prime}, N+1-j^{\prime}}\left(\lambda^{\prime}\right)}{u_{j}^{\prime}}=2 \rho
$$

The thermodynamic limit of (4.8) is computed analogously to that of (3.28). We find

$$
\begin{aligned}
& S\left(\lambda^{\prime}, \lambda ; \tau\right):=S\left(\lambda^{\prime}-\lambda ; \tau\right) \\
& \quad=\frac{1}{2} \rho^{2}\left(\int_{1}^{\infty} d u_{1} e^{-\tau\left(\pi \rho u_{1}\right)^{2} / 2 \gamma} \cos \pi u_{1} \rho\left(\lambda-\lambda^{\prime}\right) \int_{0}^{1} d u_{2} e^{\tau\left(\pi \rho u_{2}\right)^{2} / 2 \gamma} \cos \pi u_{2} \rho\left(\lambda-\lambda^{\prime}\right)\right. \\
& \quad+\int_{1}^{\infty} d u_{1} u_{1} e^{-\tau\left(\pi \rho u_{1}\right)^{2} / 2 \gamma} \sin \pi u_{1} \rho\left(\lambda-\lambda^{\prime}\right) \int_{0}^{1} d u_{2} \frac{e^{\tau\left(\pi \rho u_{2}\right)^{2} / 2 \gamma}}{u_{2}} \sin \pi u_{2} \rho\left(\lambda-\lambda^{\prime}\right)
\end{aligned}
$$

This expression is very similar to that for the orthogonal symmetry initial condition given by (3.30). In particular, apart from the factor of $1 / 2$, the first term is the density-density correlation (2.12), while the second term occurs in the expression (2.4) with $\beta_{0}=4$ for the two-point distribution. Also, the method of obtaining the large- $\left|\lambda-\lambda^{\prime}\right|, \tau$ expansion is the same as in (3.30). Behaviour corresponding to (2.13b) is verified (here it is essential that the large- $\tau$ limit also be included, for otherwise the leading- $\left|\lambda-\lambda^{\prime}\right|$ expansion is oscillatory and decays $O\left(1 /\left|\lambda-\lambda^{\prime}\right|\right)$, analogous to the $\tau=0$ result [1]).

The Fourier transform with respect to $\lambda-\lambda^{\prime}$ of (4.9) is readily calculated. We find

$$
\hat{S}(k ; \tau)=\frac{1}{2}\left(\hat{S}_{1}(k ; \tau)+\hat{S}_{3}(k ; \tau)\right)
$$

where $\hat{S}_{1}(k ; \tau)$ is given by $(3.32 \mathrm{~b})$ and

$$
\hat{S}_{3}(k ; \tau):=\frac{\gamma}{\pi \tau|k|} e^{-\pi \tau \rho|k| / \gamma} \sinh \pi \tau \rho|k| / \gamma+\frac{|k|}{2 \pi} e^{-\tau k^{2} / 2 \gamma} \int_{1-|k| / \pi \rho}^{1} d k_{1} \frac{1}{k_{1}} e^{-\pi \tau \rho|k| k_{1} / \gamma}, \quad 0 \leq|k| \leq 2 \pi \rho,
$$




$$
\hat{S}_{3}(k ; \tau):=\frac{\gamma}{\pi \tau|k|} e^{-\tau k^{2} / 2 \gamma} \sinh \pi \tau \rho|k| / \gamma+\frac{|k|}{2 \pi} e^{-\tau k^{2} / 2 \gamma} \int_{-1}^{1} d k_{1} \frac{1}{k_{1}} e^{-\pi \tau \rho|k| k_{1} / \gamma}, \quad|k| \geq 2 \pi \rho .
$$

Thus $\hat{S}(k ; \tau)$ has a logarithmic singularity at $|k|=2 \pi \rho$. The first singularity corresponds to the leading oscillatory term of the large- $\left|\lambda-\lambda^{\prime}\right|$ expansion of (4.9) which has period $1 / \pi \rho$ (the initial spacing between the doubly degenerate pairs of eigenvalues), while the second singularity corresponds to the preferred crystalline ordering of spacing $1 / 2 \pi \rho$ between eigenvalues in the final state.

For $\tau,\left|\lambda-\lambda^{\prime}\right| \rightarrow 0,(4.9)$ gives

$$
\begin{aligned}
S\left(\lambda^{\prime}-\lambda ; \tau\right) \sim & \frac{\rho}{2 \tau^{1 / 2}} \int_{0}^{\infty} d u_{1} e^{-u_{1}^{2} / 2 \gamma} \cos \pi u_{1}\left(\lambda-\lambda^{\prime}\right) / \tau^{1 / 2} \\
& +\frac{\pi \rho\left(\lambda-\lambda^{\prime}\right)}{2 \tau} \int_{0}^{\infty} d u_{1} u_{1} e^{-u_{1}^{2} / 2 \gamma} \sin \pi u_{1}\left(\lambda-\lambda^{\prime}\right) / \tau^{1 / 2} .
\end{aligned}
$$

Thus, from (2.17)

$$
\begin{aligned}
f(v) & =\frac{1}{2} \int_{0}^{\infty} d u_{1} e^{-u_{1}^{2} / 2 \gamma} \cos \pi u_{1} v+\frac{v}{2} \int_{0}^{\infty} d u_{1} u_{1} e^{-u_{1}^{2} / 2 \gamma} \sin \pi u_{1} v \\
& =\frac{1}{2}\left(\frac{\gamma}{2 \pi}\right)^{1 / 2} e^{-v^{2} \gamma / 2}\left(1+\gamma v^{2}\right)
\end{aligned}
$$

which is the arithmetic means of the distributions (2.18) and (2.20).

\section{$5 \quad$ Equispaced initial conditions}

In this section the equal parameter distribution function (2.1) will be calculated for the solution of the Dyson Brownian motion model for the circular ensemble with $\beta=2$ and the initial conditions

$$
\lambda_{j}^{(0)}=-L / 2+(j-1) / \rho+\nu, \quad 0<\nu<1 / \rho,
$$

which says the eigenvalues are all equispaced (harmonic oscillator type spectrum) at $\tau=0$.

By integrating the Green's function (3.5) over the initial configuration (5.1) (which as a distribution consists of equispaced delta function peaks), we obtain for the corresponding eigenvalue p.d.f.

$$
\begin{gathered}
p\left(\lambda_{1}, \ldots, \lambda_{N} ; \nu, \tau\right)=N^{-N / 2} e^{E_{0} \tau / \gamma} \operatorname{det}\left[\frac{1}{L} \theta_{1}\left(\pi\left(\lambda_{j}-(k-1) / \rho-\nu\right) / L ; q\right)\right]_{j, k=1, \ldots N} \\
\times \prod_{1 \leq j<k \leq N} \sin \pi\left(\lambda_{k}-\lambda_{j}\right) / L
\end{gathered}
$$

\subsection{Analogy with a classical log-potential system}

Some insight into the expected properties of the two-point equal parameter distribution for (5.2) can be obtained from a known identity [14] expressing the determinant in (5.2), averaged over $\nu$, in terms of a product:

$$
\begin{gathered}
\rho \int_{0}^{1 / \rho} d \nu \operatorname{det}\left[\frac{1}{L} \theta_{1}\left(\pi\left(\lambda_{j} / L-(k-1) / N+\nu / L\right) ; q^{1 / N}\right)\right]_{j, k=1, \ldots, N} \\
=\left(\frac{1}{L}\right)^{N} f_{N}(q) \prod_{1 \leq j<k \leq N} \theta_{1}\left(\frac{\pi}{L}\left(\lambda_{k}-\lambda_{j}\right) ; q\right)
\end{gathered}
$$


where

$$
f_{N}(q)=N^{N / 2} q^{-(N-1)(N-2) / 24}\left(\prod_{k=1}^{\infty}\left(1-q^{2 k}\right)\right)^{-(N-1)(N-2) / 2}
$$

Thus $p_{s}$ is proportional to the Jastrow-type product

$$
\prod_{1 \leq j<k \leq N} \sin \frac{\pi}{L}\left(\lambda_{k}-\lambda_{j}\right) \theta_{1}\left(\frac{\pi}{L}\left(x_{k}-x_{j}\right) ; q\right)
$$

which can be interpreted as the Boltzmann factor of a classical gas with potential energy

$$
-\sum_{1 \leq j<k \leq N} \log \left|\sin \frac{\pi}{L}\left(\lambda_{k}-\lambda_{j}\right) \theta_{1}\left(\frac{\pi}{L}\left(\lambda_{k}-\lambda_{j}\right) ; q\right)\right|
$$

and $\beta=1$.

It is well known (see e.g. [14]) that the $\theta_{1}$-function is the solution of the two-dimensional Poisson equation in doubly periodic boundary conditions, with period $L$ in the $x$-direction and period $W$ in the $y$-direction, and $q^{N}=e^{-\pi W / L}$. Furthermore, in the limit $L \rightarrow \infty$ with $W$ fixed,

$$
-\log \theta_{1}\left(\pi \lambda / L ; q^{N}\right) \sim V_{1}(\lambda) \quad \text { where } \quad V_{1}(\lambda):=-\pi|\lambda| / W
$$

while

$$
-\log \sin \pi \lambda / L \sim V_{2}(\lambda) \quad \text { where } \quad V_{2}(\lambda):=-\log |\lambda|
$$

According to Ornstein-Zernicke direct correlation function theory, the small- $k$ behaviour of the dimensionless structure factor

$$
s(k):=1+\frac{1}{\rho} \int_{-\infty}^{\infty} d x \rho_{(2)}^{T}(x) e^{i k x}
$$

of a classical gas is determined by the small- $k$ behaviour of the reciprocal of the Fourier transform of the potential:

$$
s(k) \sim \frac{1}{\beta \tilde{V}(k)} \quad \text { as } \quad k \rightarrow 0 .
$$

Now $\hat{V}_{1}(k) \sim \pi /|k|$ while $\hat{V}_{2}(k) \sim O\left(k^{2}\right)$. Hence

$$
s(k) \sim|k| / \pi
$$

or equivalently, for $\lambda \rightarrow \infty$ and $\tau$-fixed

$$
\rho_{(2)}^{T}(\lambda) \sim-\frac{1}{(\pi \lambda)^{2}}
$$

\subsection{Exact expression for the equal parameter distribution functions}

To calculate the equal parameter distributions (2.1), we multiply (5.2) by unity in the form of

$$
i^{-N(N-1) / 2} N^{N / 2} \operatorname{det}\left[e^{2 \pi i j k / N}\right]_{\substack { j=-N / 2, \ldots, N / 2-1 \\
\begin{subarray}{c}{j=1, \ldots, N \\
\text { nat. }{ j = - N / 2 , \ldots , N / 2 - 1 \\
\begin{subarray} { c } { j = 1 , \ldots , N \\
\text { nat. } } }\end{subarray}}
$$

to obtain [14],

$$
\begin{aligned}
& i^{-N(N-1) / 2} \prod_{1 \leq j<k \leq N} 2 \sin \pi\left(\lambda_{k}-\lambda_{j}\right) / L \\
& \quad \operatorname{det}\left[\frac{1}{L} e^{2 \pi i(k+1 / 2)\left(\lambda_{j}-\nu\right) / L} \theta_{3}\left(\pi \rho\left(\lambda_{j}-\nu\right)+i \pi^{2} \tau N(k+1 / 2) / L^{2} \gamma ; q^{N^{2}}\right)\right]_{\substack{k=-N / 2, \ldots, N / 2-1 \\
k=1, N, N}}^{\substack{j, N \\
k / 2}}
\end{aligned}
$$


The advantage of this expression is that with the product expressed as a Vandermonde determinant using (3.14), it consists of a product of determinants in which the elements have an orthogonality property:

$$
\int_{-L / 2}^{L / 2} d \lambda_{j} e^{-2 \pi i(k+1 / 2)\left(\lambda_{j}-\nu\right) / L} e^{2 \pi i\left(k^{\prime}+1 / 2\right)\left(\lambda_{j}-\nu\right) / L} \theta_{3}\left(\pi \rho\left(\lambda_{j}-\nu\right)+i \pi^{2} \tau N\left(k^{\prime}+1 / 2\right) / L^{2} \gamma ; q^{N^{2}}\right)=L \delta_{k, k^{\prime}}
$$

This feature allows the distributions (2.1) to be expressed as the determinant

$$
\rho_{(n)}\left(\lambda_{1}, \ldots, \lambda_{N} ; \nu, \tau\right)=\operatorname{det}\left[K\left(\lambda_{j}, \lambda_{k} ; \nu, \tau\right)\right]_{j, k=1, \ldots, N}
$$

where

$$
K\left(\lambda_{j}, \lambda_{k} ; \nu, \tau\right):=\sum_{p=-N / 2}^{N / 2-1} \frac{1}{L} e^{2 \pi i(p+1 / 2)\left(\lambda_{j}-\lambda_{k} / L\right)} \theta_{3}\left(\pi \rho\left(\lambda_{k}-\nu\right)+i \pi^{2} \tau N(p+1 / 2) / L^{2} \gamma ; e^{-2 \pi^{2} \rho^{2} \tau / \gamma}\right)
$$

The thermodynamic limit follows by noting that this expression is a Riemann sum approximation to a definite integral, which becomes exact in the thermodynamic limit, thus giving

$$
K\left(\lambda_{j}, \lambda_{k} ; \nu, \tau\right)=\rho \int_{-1 / 2}^{1 / 2} d s e^{-2 \pi i \rho\left(\lambda_{j}-\lambda_{k}\right) s} \theta_{3}\left(\pi \rho\left(\lambda_{k}-\nu\right)+i \pi^{2} \tau \rho^{2} s / \gamma ; e^{-2 \pi^{2} \rho^{2} \tau / \gamma}\right)
$$

From (5.7a) and (5.8), the truncated two-point distribution averaged over $\nu$ is given by

$$
\begin{aligned}
\rho_{(2)}^{T}\left(\lambda-\lambda^{\prime} ; \tau\right) & =-\rho^{2} \int_{-1 / 2}^{1 / 2} d s \int_{-1 / 2}^{1 / 2} d s^{\prime} e^{-2 \pi i\left(\lambda-\lambda^{\prime}\right) \rho\left(s-s^{\prime}\right)} \\
& \times \theta_{3}\left(\pi \rho\left(\lambda-\lambda^{\prime}\right)+i \pi^{2} \tau \rho^{2}\left(s-s^{\prime}\right) / \gamma ; e^{-2 \pi^{2} \rho^{2} \tau / \gamma}\right)
\end{aligned}
$$

From this expression, it is straightforward to compute the small- $|k|$ behaviour of the structure factor (5.3) (only the $n=0, \pm 1$ terms in the series definition of $\theta_{3}$ contribute). We find

$$
s(k) \sim \frac{|k|}{2 \pi}\left(1+e^{-2 \pi^{2} \tau \rho|k| / \gamma}\right)
$$

For fixed- $\tau$ the term in brackets is to leading order equal to 2 , so the result (5.4) is verified.

\subsection{Asymptotics of the spacing distribution}

The distribution $p(s)$ of the nearest neighbour spacing averaged over $\nu$ can be computed by differentiation of the probability $h(s)$ that an interval of length $s$ averaged over $\nu$ is free from eigenvalues:

$$
p(s)=-\frac{d^{2}}{d s^{2}} h(s)
$$

The leading asymptotics of $h(s)$ can be predicted by adapting an electrostatic argument of Dyson [15].

In Section 5.1 we have noted that the eigenvalue p.d.f (5.2) averaged over $\nu$ can be interpreted as the Boltzmann factor of a classical gas with potential which to leading order in large- $\lambda$ behaves as

$$
V_{1}(\lambda):=-\pi|\lambda| / W, \quad W=2 \pi \tau \rho / \gamma
$$

Now (5.11) is proportional to the potential of the Coulomb repulsion between sheets of charge (i.e. the one-dimensional Coulomb potential), and the corresponding classical gas is the one-component onedimensional plasma. The basis of the electrostatic argument is that in the macroscopic hole size limit 
$s \rightarrow \infty$, the plasma behaves like a perfect conductor. Thus the total charge $-\rho s$ inside the hole due to the uniform background will be exactly cancelled by two delta function charge distributions at each boundary of the hole of charge $\rho s / 2$.

According to the hypothesis of Dyson

$$
h(s) \sim e^{-\left(U_{1}(s)+U_{2}(s)+U_{3}(s)\right)}
$$

where $U_{1}(s), U_{2}(s)$ and $U_{3}(s)$ are given by

$$
\begin{gathered}
U_{1}(s):=-\rho^{2} \frac{\pi}{2 W} \int_{0}^{s} d x \int_{0}^{s} d y|x-y| \\
U_{2}(s):=\rho^{2} \frac{s \pi}{2 W}\left(\int_{0}^{s} d x|x|+\int_{0}^{s} d y|s-y|\right) \\
U_{3}(s):=-\left(\frac{\rho s}{2}\right)^{2} \frac{\pi s}{W}
\end{gathered}
$$

and represent the electrostatic energy of the background-background, background-boundary and boundaryboundary interactions respectively. Evaluating (5.12a) and (5.12b) and substituting in (5.11) gives

$$
h(s) \sim e^{-(\rho s)^{3} \gamma / 24 \rho^{2} \tau} .
$$

This is to be contrasted to the asymptotics of $h(s)$ with $\tau \rightarrow \infty$ (see e.g. [1]):

$$
h(s) \sim e^{-(\pi \rho s)^{2} / 8} .
$$

\section{Multiple parameters}

Beenakker and Rejaei [7] considered the multiple-parameter Fokker-Planck equation

$$
\frac{1}{d} \sum_{\mu=1}^{d} \gamma_{\mu} \frac{\partial P}{\partial \tau_{\nu}}=\mathcal{L} P
$$

with $\mathcal{L}$ given by (1.1) and $W$ by (1.2), as a model for the statistical description of the dispersion relation of a $d$-dimensional crystalline lattice. With the initial condition (2.7) extended to $\vec{\tau}=\overrightarrow{0}$, it is easy to see that the Green's function solution of (6.1) with $\beta=2, N$ odd and $W$ given by (3.1) is obtained by making the replacement

$$
\frac{1}{L} \theta_{3}\left(\pi\left(\lambda_{j}-\lambda_{k}\right) / L ; q\right) \mapsto \frac{1}{d} \sum_{\mu=1}^{d} \theta_{3}\left(\pi\left(\lambda_{j}-\lambda_{k}\right) / L ; q_{\mu}\right), \quad q_{\mu}:=e^{-2 \pi^{2} \tau_{\mu} / L^{2} \gamma_{\mu}}
$$

Furthermore, explicit calculation shows that the density-density correlations and equal-time correlations for the multiple-parameter equation are the same as for the single-parameter equation (with corresponding initial eigenvalue p.d.f.'s) provided the replacements

$$
e^{ \pm \pi^{2} \rho^{2} u^{2} \tau / 2 \gamma} \mapsto\left(\frac{1}{d} \sum_{\mu=1}^{d} e^{-\pi^{2} \rho^{2} u^{2} \tau_{\mu} / 2 \gamma_{\mu}}\right)^{\mp 1}
$$

are made in each of the integrands of (2.11), (3.32) and (4.9), while in (5.7) the replacement

$$
\theta_{3}\left(\pi \rho\left(\lambda_{k}-\nu\right)+i \pi^{2} \tau s \rho^{2} / \gamma ; e^{-2 \pi^{2} \rho^{2} \tau / \gamma}\right) \mapsto \frac{1}{d} \sum_{\mu=1}^{d} \theta_{3}\left(\pi \rho\left(\lambda_{k}-\nu\right)+i \pi^{2} \tau_{\mu} s \rho^{2} / \gamma_{\mu} ; e^{-2 \pi^{2} \rho^{2} \tau_{\mu} / \gamma_{\mu}}\right)
$$


is required.

As an example, the multiple parameter generalization of the density-density correlation (2.11) for initial conditions with unitary symmetry is

$$
\begin{gathered}
S\left(\lambda-\lambda^{\prime} ; \vec{\tau}\right)=\rho^{2} \int_{1}^{\infty} d u_{1}\left(\frac{1}{d} \sum_{\mu=1}^{d} e^{-\pi^{2} \rho^{2} u_{1}^{2} \tau_{\mu} / 2 \gamma_{\mu}}\right) \cos \pi u_{1} \rho\left(\lambda-\lambda^{\prime}\right) \\
\times \int_{0}^{1} d u_{2}\left(\frac{1}{d} \sum_{\mu=1}^{d} e^{-\pi^{2} \rho^{2} u_{2}^{2} \tau_{\mu} / 2 \gamma_{\mu}}\right)^{-1} \cos \pi u_{2} \rho\left(\lambda-\lambda^{\prime}\right)
\end{gathered}
$$

For large- $\left|\lambda-\lambda^{\prime}\right|$ and $\tau_{\mu}(\mu=1, \ldots, d)$ (with each $\tau_{\mu}$ of the same order), the asymptotic expansion of (6.5) is obtained by writing

$$
\begin{aligned}
\sum_{\mu=1}^{d} e^{-\pi^{2} \rho^{2} u_{2}^{2} \tau_{\mu} / 2 \gamma_{\mu}}=\exp \left(-\frac{1}{d} \sum_{\mu=1}^{d} \frac{\tau_{\mu}}{2 \gamma_{\mu}}\left(\pi \rho u_{2}\right)^{2}\right) \\
\times \sum_{\mu=1}^{d} \exp \left(-\left(\frac{d-1}{d} \frac{\tau_{\mu}}{2 \gamma_{\mu}}-\frac{1}{d} \sum_{\substack{\sigma=1 \\
\sigma \neq \mu}}^{d} \frac{\tau_{\sigma}}{2 \gamma_{\sigma}}\right)\left(\pi \rho u_{2}\right)^{2}\right)
\end{aligned}
$$

By the assumption on the relative order of each $\tau_{\mu}$, we see the second term on the r.h.s. is $O(1)$ and can so be ignored for purposes of computing the leading order asymptotic behaviour. Thus we again find the formula (2.10) with $\beta=2$, provided the replacement

$$
\frac{\tau}{2 \gamma} \mapsto \frac{1}{d} \sum_{\mu=1}^{d} \frac{\tau_{\mu}}{2 \gamma_{\mu}}
$$

is made. This same prescription gives the asymptotic expansion of the density-density correlation with multiple parameters for orthogonal and symplectic symmetry from the corresponding expansion for the single parameter case obtained in the text. These finding are in agreement with those in [7] (however the requirement that the expansions are only valid for each $\tau_{\mu}$ of the same order was not made explicit in $[7])$.

Similarly, we find the distributions (2.17), (2.19), (3.33) and (4.11) are unchanged, provided the replacement

$$
\left(\frac{\gamma}{2 \pi}\right)^{1 / 2} e^{-v^{2} \gamma / 2} \mapsto \frac{1}{d} \sum_{\mu=1}^{d}\left(\frac{\gamma_{\mu}}{2 \pi}\right)^{1 / 2} e^{-v^{2} \gamma_{\mu} / 2}
$$

is made.

Note added: Since the completion of this work, I have been informed by J.A. Zuk that the results of ref. [3] include the calculation of the density-density correlations given in Sections 3 and 4. However these results, which are derived using the supersymmetry method, are presented as complicated triple integrals, whereas our results (3.30) and (4.9) are products of simple single integrals. We have not been able to show directly that the different forms are equivalent. Instead Zuk [16] is repeating the calculation of [3], with a different parametrization [17] which is known to reproduce the equal parameter results (2.4)-(2.6), in a bid to derive (3.30) and (4.9) using the supersymmetry method. He also points out that taking the 
Laplace transform of (3.32) in the variable $t:=(\pi \rho)^{2} \tau / 2 \gamma$ gives a result entirely in terms of elementary functions:

$$
\begin{aligned}
\tilde{S}(k, s) & :=\int_{-\infty}^{\infty} d x e^{i k x} \int_{0}^{\infty} d t e^{-s t} \rho^{-2} S(\lambda ; \tau), \quad(x:=\pi \rho \lambda) \\
& =\frac{\pi}{2|k|\left(s-k^{2}\right)}\left\{\begin{array}{l}
s \log \frac{(1+|k|)^{2}+s-1}{-(1-|k|)^{2}+s+1}-k^{2} \log (1+|k|), \quad|k| \leq 2 \\
s \log \frac{(|k|+1)^{2}+s-1}{(|k|-1)^{2}+s-1}-k^{2} \log \frac{|k|+1}{|k|-1}, \quad|k| \geq 2
\end{array}\right.
\end{aligned}
$$

We also note that the paper [18] has appeared which states a result (eq. (47)) equivalent to our (5.9). 


\section{References}

[1] M.L. Mehta, Random Matrices, 2nd ed. (Academic, New York, 1991)

[2] Z. Pluhař, H.A. Weidenmüller, J.A. Zuk and C.H. Lewenkopf, Phys. Rev. Lett. 73, 2155 (1994)

[3] N. Taniguchi, A. Hashimoto, B.D. Simons and B.L. Altshuler, Europhys. Lett. 27, 335 (1994)

[4] F. Haake, Quantum Signatures of Chaos (Springer, Berlin, 1992)

[5] A. Pandey and M.L. Mehta, Comm. Math. Phys. 87, 449 (1983); M.L. Mehta and A. Pandey, J. Phys. A 16, 2655 (1983)

[6] A. Pandey and P. Shulka, J. Phys. A 24, 3907 (1991)

[7] C.W.J. Beenakker and B. Rejaei, Physica A 203, 61 (1994)

[8] A.M.S. Macêdo, Europhys. Lett. 27, 335 (1994)

[9] V.E. Kravstov and M.R. Zirnbauer, Phys. Rev. B 46, 4332 (1992)

[10] B.D. Simons and B.L. Altshuler, Phys. Rev. B 48, 5422 (1993)

[11] F.J. Dyson, J. Math. Phys. 3, 1199 (1962)

[12] H. Risken, The Fokker Planck equation, (Springer, Berlin, 1992)

[13] P.J. Forrester, J. Phys. A 23, 1259 (1990)

[14] P.J. Forrester, SIAM J. Math. Anal. 21, 270 (1990)

[15] F.J. Dyson, J. Math. Phys. 3, 157 (1962)

[16] J.A. Zuk, in preparation

[17] A. Altland, S. Iida and K.B. Efetov, J. Phys. A 26, 3545 (1993)

[18] A. Pandey, Chaos, Solitons and Fractals 5, 1275 (1995) 